\title{
MODIFIABLE RISK FACTORS ASSOCIATED WITH DEEP STERNAL SITE INFECTION AFTER CORONARY ARTERY BYPASS GRAFTING
}

William E. Trick, MD

William E. Scheckler, MD

Jerome I. Tokars, MD ${ }^{\text {a }}$

Kevin C. Jones, $\mathrm{DO}^{\mathrm{a}}$

Mel L. Reppen, $\mathrm{RN}^{\mathrm{b}}$

Ellen M. Smith, RN ${ }^{b}$

William R. Jarvis, MD
Objective: Our objective was to identify risk factors for deep sternal site infection after coronary artery bypass grafting at a community hospital. Methods: We compared the prevalence of deep sternal site infection among patients having coronary artery bypass grafting during the study (January 1995-March 1998) and pre-study (January 1992-December 1994) periods. We compared any patient having a deep sternal site infection after coronary artery bypass graft surgery during the study period (case-patients) with randomly selected patients who had coronary artery bypass graft surgery but no deep sternal site infection during the same period (control-patients). Results: Deep sternal site infections were significantly more common during the study than during the pre-study period (30/1796 [1.7\%] vs 9/1232 [0.7\%]; $P=.04)$. Among 30 casepatients, $29(97 \%)$ returned to the operating room for sternal debridement or rewiring, and $2(7 \%)$ died. In multivariable analyses, cefuroxime receipt 2 hours or more before incision (odds ratio $=5.0$ ), diabetes mellitus with a preoperative blood glucose level of $200 \mathrm{mg} / \mathrm{dL}$ or more $($ odds ratio $=\mathbf{1 0 . 2})$, and staple use for skin closure $($ odds ratio $=4.0$ ) were independent risk factors for deep sternal site infection. Staple use was a risk factor only for patients with a normal body mass index. Conclusions: Appropriate timing of antimicrobial prophylaxis, control of preoperative blood glucose levels, and avoidance of staple use in patients with a normal body mass index should prevent deep sternal site infection after coronary artery bypass graft operations. (J Thorac Cardiovasc Surg 2000;119:108-14)
In the United States, more than 450,000 coronary artery bypass graft $(\mathrm{CABG})$ procedures are performed each year. ${ }^{1}$ Deep sternal site infection (DSSI) is a complication of CABG surgery associated with substantial morbidity and with mortality as high as $14 \% .^{2,3}$ In addition, a DSSI has been estimated to increase the cost of each operation nearly 3 -fold. ${ }^{2}$ Several recent reports document DSSI prevalence between $0.7 \%$ and $3.9 \% .^{2,4-9}$ Because of high patient morbidity and mortality associated with post-CABG DSSI and the finan-

From the Hospital Infections Program, Centers for Disease Control and Prevention, Atlanta, $\mathrm{Ga},{ }^{\mathrm{a}}$ and Saint Marys Hospital Medical Center, Madison, Wis. ${ }^{\mathrm{b}}$

Received for publication May 21, 1999; revisions requested July 16, 1999; revisions received Aug 13, 1999; accepted for publication Sept 21, 1999.

Address for reprints: William E. Trick, MD, Hospital Infections Program/CDC, 1600 Clifton Rd, MS E-69, Atlanta, GA 30333 (E-mail: wbt9@cdc.gov).

Copyright @ 2000 by Mosby, Inc.

$0022-5223 / 2000 \$ 12.00+0 \quad \mathbf{1 2 / 1 / 1 0 3 1 5 4}$ cial cost to an institution from unreimbursed charges, there is great interest in identifying ways to minimize these infections. Interventions should target modifiable surgical or patient risk factors and infection control practices. Several reports have described risk factors for DSSI after median sternotomy. Some of the host risk factors identified include sex,${ }^{4,6}$ age, ${ }^{4,10}$ obesity, ${ }^{2-4,8,11,12}$ poor functional cardiac status, ${ }^{10}$ low cardiac output state, ${ }^{9}$ diabetes mellitus, ${ }^{3,5,12}$ elevated postoperative blood glucose levels, ${ }^{8}$ and preoperative Staphylococcus aureus nasal carriage. ${ }^{13}$ Surgical risk factors identified include prolonged duration of the operation, ${ }^{2,9}$ postoperative bleeding, ${ }^{9,14}$ repeat cardiac operation,,${ }^{9,14,15}$ bilateral internal thoracic artery harvest, ${ }^{2,11}$ and indiscriminate use of electrocautery. ${ }^{16}$ Despite identification of risk factors for infection, modification of many of these risk factors can be difficult or impossible. However, intervention is possible in some host and surgical risk factors, and some infection control principles are available that should minimize bacterial contamination during the operation. 


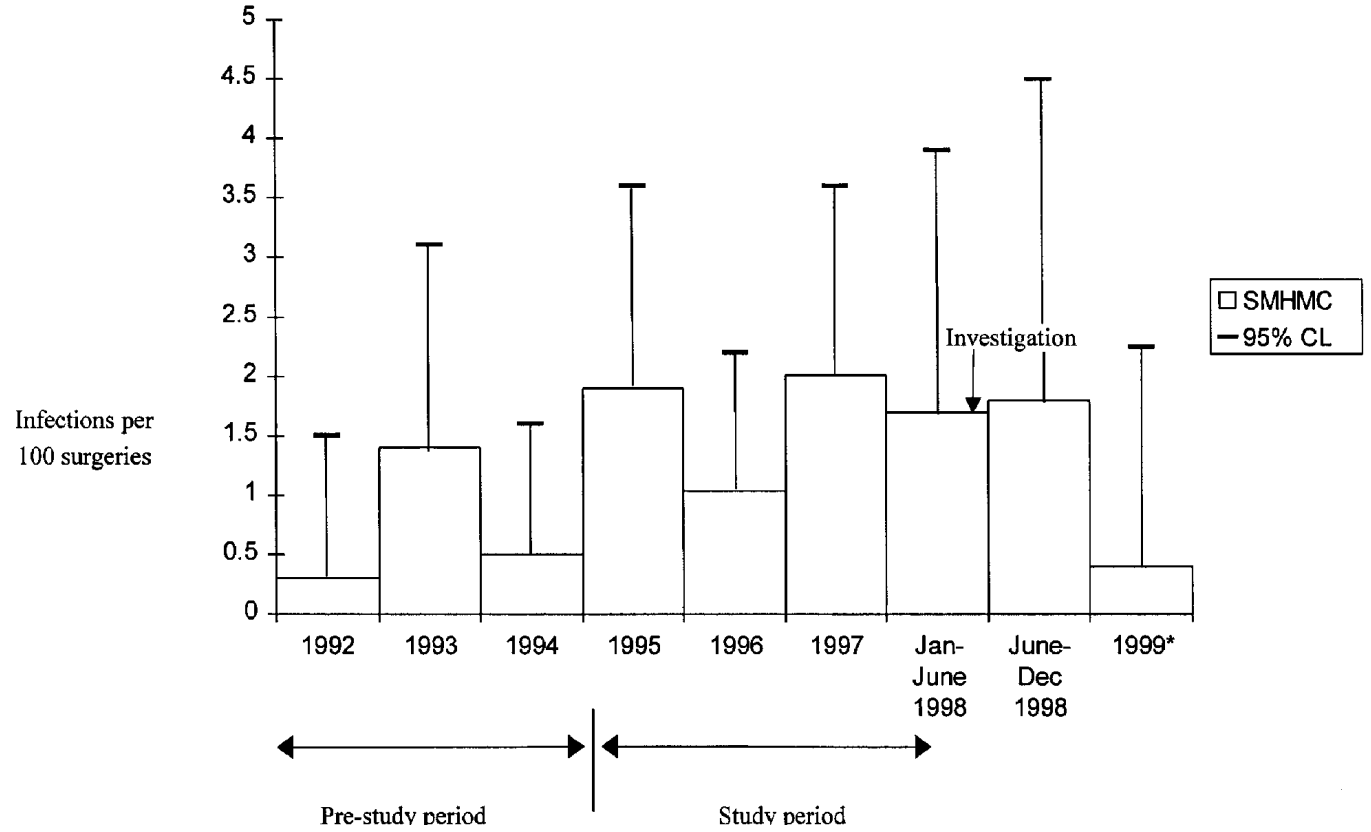

Fig 1. Prevalence of post-CABG DSSI at St Marys Hospital Medical Center (SMHMC), Madison, Wisconsin, January 1, 1992, through March 31, 1999. *Data were available for the first two quarters of 1999.

From January 1995 through March 1998 (study period), personnel at Saint Marys Hospital Medical Center (SMHMC), Madison, Wisconsin, detected an increasing prevalence of post-CABG DSSI, that is, sternal osteomyelitis or mediastinitis (Fig 1). In addition, the DSSI prevalence during the study period at SMHMC (1.7\%) was higher than the Centers for Disease Control and Prevention's (CDC) National Nosocomial Infections Surveillance (NNIS) system pooled mean rate for 1992 through 1997 of $0.65 \%$. Despite efforts at SMHMC to reduce the DSSI prevalence, the prevalence remained higher than the NNIS system rate. To formulate an intervention to prevent post-CABG DSSI, we evaluated CABG procedures at SMHMC, collected data on all measurable, previously reported risk factors, and selected potential risk factors not previously reported.

\section{Patients and methods}

Background. SMHMC is a 350-bed community hospital. From 1995 through 1997, cardiothoracic surgeons at SMHMC performed approximately 530 CABG procedures per year. The majority of operations were performed in one of three operating rooms; two of these are used almost exclusively for $\mathrm{CABG}$ procedures, and the third operating room also was used for other vascular procedures. There was no cardiothoracic house staff at SMHMC, and all of the cardiothoracic surgeons operated exclusively at SMHMC.

Case definition and study period. A case-patient was defined as any patient who had undergone a CABG procedure at SMHMC during the study period and subsequently had a deep incisional or organ/space DSSI as defined by the CDC. ${ }^{17}$ To verify that the post-CABG DSSI prevalence had significantly increased, we compared the post-CABG DSSI prevalence (ie, the number of post-CABG DSSIs divided by the total number of CABG procedures performed by median sternotomy) in the study and pre-study periods (January 1992 through December 1994.

Case ascertainment and control selection. All casepatients had been identified during surveillance by infection control personnel at SMHMC. Surveillance at SMHMC included regular review of inpatients who had undergone a CABG procedure, review of microbiology laboratory culture results, notification of infection control practitioners at SMHMC by an outpatient clinic physician or nurse, and evaluation of patients readmitted after CABG operations. Medical records of case-patients were reviewed to verify that the case definition criteria were met. Control-patients $(\mathrm{n}=$ 90) were randomly selected in a 3:1 ratio from the population of patients who had a CABG procedure performed by median sternotomy during the study period but did not acquire a DSSI.

Case-control study. To identify exposures associated with DSSI, we reviewed the medical records of case-patients and control-patients. Preoperative variables examined included the following: American Society of Anesthesiologists (ASA) classification; blood glucose level (obtained within 24 hours before the operation); body mass index [BMI (kilograms per square meter)]; diagnosis; duration of hospitalization; left 
Table I. Comparison of risk factors among case-patients and control-patients at Saint Marys Hospital Medical Center, Madison, Wisconsin, January 1995 through March 1998

\begin{tabular}{|c|c|c|c|c|}
\hline Variable & $\begin{array}{l}\text { Case-patients } \\
\quad(n=30)\end{array}$ & $\begin{array}{l}\text { Control-patients } \\
\quad(n=90)\end{array}$ & $\begin{array}{l}\text { Odds ratio } \\
(95 \% \text { CI })\end{array}$ & $\mathrm{P}$ value \\
\hline \multicolumn{5}{|l|}{ Categoric variables, No. (\%) } \\
\hline Staples for chest skin closure & $18(60)$ & $29(32)$ & $3.2(1.2-8.2)$ & .007 \\
\hline Bilateral ITA graft & $4(13)$ & $1(1)$ & $14(1.3-342)$ & .01 \\
\hline Diabetes mellitus receiving insulin & $8(27)$ & $8(8)$ & $3.7(1.1-13)$ & .01 \\
\hline Diabetes mellitus & $17(57)$ & $30(33)$ & $2.6(1.0-6.7)$ & .02 \\
\hline Preoperative glucose $\geq 200 \mathrm{mg} / \mathrm{dL}^{*}$ & $9 / 15(60)$ & $6 / 26(23)$ & $5.0(1.0-26)$ & .02 \\
\hline $\begin{array}{l}\text { Cefuroxime receipt } \geq 2 \mathrm{~h} \text { before incision or } \\
\text { after operation }^{* \dagger}\end{array}$ & $14 / 24(58)$ & $23 / 72(32)$ & $3.0(1.0-8.7)$ & .02 \\
\hline Cefuroxime receipt $\geq 2 \mathrm{~h}$ before incision ${ }^{* \dagger}$ & $12 / 22(55)$ & $20 / 69(29)$ & $2.9(1.0-9.0)$ & .03 \\
\hline $\begin{array}{l}\text { Antimicrobial receipt } \geq 2 \mathrm{~h} \text { before incision or } \\
\text { after operation }\end{array}$ & $16 / 28(57)$ & 31/87 (36) & $2.4(0.9-6.3)$ & .04 \\
\hline COPD & $4(13)$ & $5(6)$ & $2.6(0.5-13)$ & .16 \\
\hline ASA score $>3$ & $19(63)$ & $44(49)$ & $1.8(0.7-4.6)$ & .17 \\
\hline Obese $(\mathrm{BMI} \geq 30)^{*}$ & $13 / 29(45)$ & $26 / 84(31)$ & $1.8(0.7-4.8)$ & .18 \\
\hline Chest tube removed after POD 2 & $16(53)$ & $36(40)$ & $1.7(0.7-4.3)$ & .20 \\
\hline Repeat CABG & $5(17)$ & $9(10)$ & $1.8(0.5-6.7)$ & .32 \\
\hline $\mathrm{LVEF} \leq 40 \%{ }^{*}$ & $7 / 29(24)$ & $13 / 82(16)$ & $1.7(0.5-5.4)$ & .32 \\
\hline Hospitalization before procedure & $18(60)$ & $58(64)$ & $0.8(0.3-2.1)$ & .83 \\
\hline Another procedure concurrent with CABG & $3(10)$ & $10(11)$ & $0.9(0.2-3.9)$ & 1.0 \\
\hline \multicolumn{5}{|l|}{ Continuous median (interquartile-range) } \\
\hline Pulmonary artery diastolic pressure $(\mathrm{mm} \mathrm{Hg})$ & \multicolumn{2}{|c|}{$20(17-26)[n=27]$} & {$[\mathrm{n}=81]$} & .01 \\
\hline Glucose level before operation (mg/dL) & $225(158-275)[n=15]$ & $158(12$ & $158(121-180)[\mathrm{n}=26]$ & .02 \\
\hline Glucose level after operation (mg/dL) & \multicolumn{2}{|c|}{$262(241-295)[\mathrm{n}=17]$} & $244(208-274)[n=30]$ & .04 \\
\hline Duration of hypothermia (min) & $70(45-92)$ & \multicolumn{2}{|c|}{$59(35-87)$} & .34 \\
\hline Volume in cell salvage system (mL) & $955(800-1200)$ & \multicolumn{2}{|c|}{$900(810-1125)$} & .57 \\
\hline Preoperative hospitalization (d) & $3.0(1.0-6.0)$ & \multicolumn{2}{|c|}{$4.0(2.0-6.0)$} & .72 \\
\hline Age (y) & $66(59-72)$ & \multicolumn{2}{|c|}{$65(58-73)$} & .87 \\
\hline Clamp time (min) & $81(63$ & $86(69$ & & .87 \\
\hline Pump time (min) & $119(94$ & $120(9$ & & .89 \\
\hline No. of CABGs & 3.0( & 3.0( & & .92 \\
\hline Duration of operation (min) & $252(22$ & $244(20$ & & .95 \\
\hline TISS score & $35(35$ & 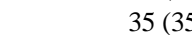 & & .99 \\
\hline
\end{tabular}

$C I$, Confidence interval; ITA, internal thoracic artery; $C O P D$, chronic obstructive pulmonary disease; $A S A$, American Society of Anesthesiology; $B M I$, body mass index; $P O D$, postoperative day; $C A B G$, coronary artery bypass graft; $L V E F$, left ventricular ejection fraction; TISS, Therapeutic Intervention Scoring System.

"Patients for whom data were missing were excluded from these analyses. Denominators are displayed for these variables.

Includes only patients who received cefuroxime.

ventricular ejection fraction; steroid receipt (as recorded in the preoperative assessment); tobacco use; and urgency of the procedure (elective, urgent, or emergency). Intraoperative variables examined included the following: associated procedure(s); volume of blood retrieved from the cell salvage system; day of the week on which the procedure was performed; duration of hypothermia (temperature $<35^{\circ} \mathrm{C}$ ); NNIS surgical risk index; operating room number; operating room personnel; perfusion, pump, and aortic crossclamp times (minutes); prophylactic antimicrobial agent administered and the timing and duration of administration; source and number of grafts; temporary pacemaker placement; and type of skin closure (ie, staple or suture closure). The following postoperative variables were examined: antimicrobial use; blood glucose level (average of peak glucose levels for the first 7 days after the operation); duration of chest tube placement; dura- tion of prophylactic antimicrobial receipt; number of red blood cell units transfused; severity of illness 24 hours after the operation, as measured by the Therapeutic Intervention Scoring System (TISS) ${ }^{18}$; and volume of chest tube output.

Procedure review/observational studies. We reviewed the preoperative skin preparation protocol and practice on inpatients and early morning admissions. We observed operating room skin preparation and patient draping. To monitor for breaks in sterile technique, we observed several CABG procedures, including saphenous vein, internal thoracic artery, and radial artery graft harvests. We toured the surgical intensive care unit and reviewed protocols for incision site care.

Statistical methods. Data were collected on a standardized form, entered, and analyzed with the CDC's Epi Info version 6.03 software. We analyzed several continuous variables after creating dichotomous variables using the following cutoff 
values: BMI more than $30 \mathrm{~kg} / \mathrm{m}^{2}$, blood glucose level 200 $\mathrm{mg} / \mathrm{dL}$ or more, left ventricular ejection fraction more than $40 \%$, and initiation of cefuroxime antimicrobial prophylaxis 120 minutes or more before skin incision. During logistic regression analyses, patients were classified into one of the following mutually exclusive categories: no diagnosis of diabetes mellitus, diabetes mellitus with a preoperative blood glucose level less than $200 \mathrm{mg} / \mathrm{dL}$, and diabetes mellitus with a preoperative blood glucose level of $200 \mathrm{mg} / \mathrm{dL}$ or more.

Categoric variables were compared with the use of either the Fisher exact test or $\chi^{2}$ test. Odds ratios and $95 \%$ confidence intervals were calculated. Continuous variables were compared with the use of the Kruskal-Wallis test for nonparametric data. Mantel-Haenszel summary odds ratios and 95\% confidence intervals were calculated for all stratified analyses. To identify independent risk factors, we constructed a logistic regression model using SAS for personal computers (SAS Institute, Cary, NC). All variables with $P<.1$ by univariate analysis were evaluated by stepwise logistic regression for inclusion in the final model.

\section{Results}

DSSI prevalence and case-patient characteristics. The DSSI prevalence was significantly higher in the study than in the pre-study period $(30 / 1796[1.7 \%]$ vs 9/1232 [0.7\%]; $P=.04$ ). Thirty patients met the case definition. Case-patients had a median age of 66 years (range 59-72 years), 22 (73\%) were male, 17 (57\%) had diabetes mellitus, 13 (43\%) were obese, and 29 (97\%) returned to the operating room for sternal rewiring or sternal debridement. The median incubation period was 15 days (range 7-161 days). In 10 casepatients (33\%) DSSI was diagnosed during the same admission in which the CABG operation was performed, and in 20 (67\%) the diagnosis was made after readmission to the hospital. The median duration of hospitalization for patients readmitted with DSSI was 16 days (range 4-74 days). Twelve (40\%) case-patients required sternal reconstruction with muscle flaps, and 2 (6.7\%) died during the hospitalization for DSSI. Culture-positive specimens were obtained from the DSSI in 27 case-patients; pathogens included Staphylococcus epidermidis $(\mathrm{n}=12,44 \%), S$. aureus $(\mathrm{n}=4$, $15 \%)$, Pseudomonas aeruginosa $(\mathrm{n}=2,7 \%)$, enterococcus $(\mathrm{n}=1,4 \%)$, and polymicrobial infections $(\mathrm{n}=$ $8,30 \%$ ). In 3 case-patients no organism was isolated.

Case-control study. The median duration of postoperative hospitalization for case-patients in whom DSSI was diagnosed during the surgery admission $(n=10)$ was significantly longer than the duration for 90 control-patients: 28 versus 6 days $(P<.001)$. In univariate analysis, case-patients and control-patients were similar with regard to the following criteria: age; ASA clas-
Table II. Independent risk factors for post-CABG DSSI at Saint Marys Hospital Medical Center, Madison, Wisconsin, January 1, 1995, through March 31, 1998

\begin{tabular}{lll}
\hline Variable & $\begin{array}{c}\text { Odds ratio } \\
(95 \% \mathrm{CI})\end{array}$ & P value \\
\hline Staples used for chest skin closure & & \\
$\quad$ No & 1.0 & - \\
$\quad$ Yes & $4.0(1.4-12)$ & .01 \\
Diabetes mellitus & & \\
No & 1.0 & - \\
Yes, preoperative glucose $<200 \mathrm{mg} / \mathrm{dL}$ & $1.4(0.4-4.8)$ & .6 \\
$\quad$ Yes, preoperative glucose $\geq 200 \mathrm{mg} / \mathrm{dL}$ & $10.2(2.4-43)$ & .008 \\
Antimicrobial receipt & & \\
$\quad$ Cefuroxime $<2 \mathrm{~h}$ before incision & 1.0 & - \\
$\quad$ Cefuroxime $\geq 2 \mathrm{~h}$ before incision & $5.0(1.4-17)$ & .002 \\
Vancomycin receipt & $4.2(0.9-20)$ & .07 \\
\hline
\end{tabular}

CI, Confidence interval.

sification; sex; hospitalization before the operation; duration of preoperative stay; NNIS risk index; repeat CABG; type of graft (except bilateral internal thoracic artery); number of grafts; placement of temporary pacemaker; duration of chest tube placement; operating room; incision time; obesity; surgeon; duration of operation, pump or clamp time, and hypothermia; volume from the cell salvage system; TISS score; and volume of chest tube output. In contrast, case-patients were significantly $(P<0.05)$ more likely than control-patients to have had bilateral internal thoracic artery harvest, staples used for skin closure, diabetes mellitus (with and without insulin use), glucose level of $200 \mathrm{mg} / \mathrm{dL}$ or more before the operation, higher blood glucose levels before and after the operation, and a higher pulmonary artery diastolic pressure (Table I).

Of 119 patients who had documented antimicrobial prophylaxis, all received antimicrobial prophylaxis with either cefuroxime $(n=100)$ or vancomycin $(n=$ 19). Receipt of the prophylactic antimicrobial agent 120 minutes or more before skin incision was significantly associated with DSSI. Since cefuroxime and vancomycin have different pharmaceutical half-lives, we also evaluated timing of antimicrobial receipt for the subset of patients who had received cefuroxime as the prophylactic antimicrobial agent. Receipt of cefuroxime 120 minutes or more before skin incision was significantly associated with DSSI (Table I).

We present two final logistic regression models (Tables II and III). Model 1 (Table II) included significant variables identified in the stepwise logistic regression analysis: staple use for skin closure, diabetes mellitus categorized as described in the "Patients and methods" section, and prophylactic antimicrobial agent 
Table III. Evaluation of the interaction between staple use for skin closure and body mass index (BMI), ${ }^{*}$ Saint Marys Hospital Medical Center, Madison, Wisconsin, January 1, 1995, through March 31, 1998

\begin{tabular}{llcccc}
\hline Staples & BMI & $\begin{array}{c}\text { Case-patients }^{\ddagger} \\
{[n=29](\%)}\end{array}$ & $\begin{array}{c}\text { Control-patients }^{\ddagger} \\
{[n=85](\%)}\end{array}$ & $\begin{array}{c}\text { Odds ratio }^{\S} \\
(95 \% \text { CI) }\end{array}$ & P value \\
\hline No & Normal & $4(14)$ & $42(49)$ & 1.0 & - \\
No & High & $8(28)$ & $16(19)$ & $4.8(0.8-30)$ & .1 \\
Yes & High & $5(17)$ & $10(12)$ & $4.4(0.6-34)$ & .15 \\
Yes & Normal & $12(41)$ & $17(20)$ & $13.5(2.3-79)$ & .004 \\
\hline
\end{tabular}

*The interaction of staples used for skin closure and BMI was statistically significant.

'High BMI, > $30 \mathrm{~kg} / \mathrm{m}^{2}$; normal BMI, $\leq 30 \mathrm{~kg} / \mathrm{m}^{2}$.

Case-patients and control-patients who had BMI recorded $(\mathrm{n}=114)$.

${ }^{\S}$ Values determined in a logistic regression model that included diabetes mellitus and antimicrobial receipt (as per Table II).

and timing of administration. Independent risk factors identified by multivariable analysis were skin closure with staples, diabetes mellitus with a preoperative glucose level of $200 \mathrm{mg} / \mathrm{dL}$ or more, or cefuroxime receipt 2 hours or more before the operation (Table II). Diabetes mellitus with a preoperative blood glucose level less than $200 \mathrm{mg} / \mathrm{dL}$ was not associated with DSSI.

Because staple use for skin closure has not previously been identified as a risk factor for post-CABG DSSI, we attempted to identify confounding variables. We performed stratified analyses with staple use and multiple previously identified risk factors (eg, duration of the operation, urgency of the procedure, BMI, and ASA classification). The interaction of staple use and BMI was statistically significant $(P=.03)$. We used logistic regression (model 2) to evaluate staple use in patients stratified by BMI. Model 2 also included diabetes mellitus and antimicrobial receipt as categorized in model 1. Staple use for skin closure was associated with DSSI only in patients who had a normal BMI (Table III).

Skin preparation procedure review. For inpatients, the skin preparation protocol before CABG surgery included an antiseptic scrub, suppository insertion, hair removal by clipping, and a repeat antiseptic scrub, all performed the night before the operation. No repeat morning scrub was administered. For patients admitted the same day as the operation, a similar protocol was followed but performed the morning of the operation. For the majority of patients, hair removal by clipping was performed, regardless of sex.

\section{Discussion}

Using CDC surveillance methods, personnel at SMHMC identified a high prevalence of post-CABG DSSI. A detailed investigation revealed three independent risk factors, all of which are potentially modifiable: preoperative hyperglycemia, administration of cefuroxime prophylaxis 2 hours or more before skin incision, and skin closure with staples among patients with a normal BMI.

Diabetes mellitus ${ }^{3,5,12}$ and elevated postoperative blood glucose levels ${ }^{8}$ have been identified as risk factors for DSSI. Our data suggest that diabetes mellitus is a risk factor only when the preoperative blood glucose level is $200 \mathrm{mg} / \mathrm{dL}$ or higher. Elevated blood glucose levels (eg, glucose $>200 \mathrm{mg} / \mathrm{dL}$ ) have been associated with increased infection among persons with diabetes, ${ }^{19}$ and specific detrimental effects of such levels on the host immune system have been described. ${ }^{20}$ Optimal immune system function at the time of the operation, when risk of contamination is increased, may be critical to prevention of DSSI. Improvements in preoperative metabolic factors (eg, blood glucose levels) are necessary to optimize immune system function. There is evidence that improved glucose control in patients with diabetes may improve immune system function, ${ }^{21}$ and one study reported a decrease in DSSI after initiation of a protocol to reduce postoperative blood glucose levels to less than $200 \mathrm{mg} / \mathrm{dL} .{ }^{8}$ An alternative hypothesis is that prolonged elevation of glucose results in end organ damage that may be associated with increased risk for DSSI. Since suboptimal glycemic control has been correlated with end organ dysfunction, ${ }^{22,23}$ it may be difficult to determine the relative contribution of hyperglycemia in the immediate preoperative period versus the detrimental effects of prolonged hyperglycemia. One possibility would be to compare glycosylated hemoglobin values, end organ dysfunction (eg, retinopathy, nephropathy, or neuropathy), and blood glucose levels before the operation as risk factors for DSSI. Unfortunately, in our study, glycosylated hemoglobin values were not available for the majority of patients. To our knowledge, improved glucose control before surgery has not been shown to decrease the risk of infection after the operation; however, because of the association of increased blood glu- 
cose levels and infection and the probability of improved immune system function with a decrease in blood glucose level, it is prudent to lower blood glucose levels $(<200 \mathrm{mg} / \mathrm{dL})$ in patients about to undergo CABG operations.

Although the optimal time for preoperative receipt of antimicrobial prophylaxis among patients about to undergo varied surgical procedures has been described ${ }^{24}$ we know of no reports that confirm this finding in the CABG surgery population. Cefuroxime has a shorter pharmaceutical half-life than vancomycin; therefore, early dosing of cefuroxime may result in relatively low blood and tissue concentrations at the time of surgery. Appropriate timing of administration likely is more critical if cefuroxime is given as the prophylactic antimicrobial agent.

We analyzed timing of cefuroxime receipt independent of vancomycin receipt. We compared cefuroxime receipt 2 hours or more before the operation (early antimicrobial prophylaxis) with cefuroxime receipt less than 2 hours before incision. We found that those patients receiving early cefuroxime antimicrobial prophylaxis had an increased risk of DSSI. Vancomycin receipt was associated with DSSI, but the result may have been due to chance. In addition, we included vancomycin in our analysis as a single risk factor regardless of "appropriate" or "inappropriate" timing of administration. Since several patients received vancomycin 120 minutes or more before skin incision, we cannot make any conclusions about vancomycin and cefuroxime as prophylactic antimicrobial agents before CABG operations.

Staple use for skin closure has not previously been reported as a risk factor for post-CABG DSSI. In our multivariable analysis controlling for potential confounders, we found that staple use was a risk factor only in patients with a normal BMI. One possible explanation is that staple depth in non-obese patients in a region with relatively little subcutaneous tissue may increase the possibility of microbial invasion to anatomically deeper tissue planes, resulting in DSSI. We acknowledge that a retrospective study design is suboptimal for determining causality and that unmeasured characteristics may have influenced these results. However, after literature review, we collected data on all previously reported measurable risk factors. In addition, contrary to previous studies, we found no association between obesity and DSSI.

Limitations of this investigation included the inability to obtain complete data for all variables. Specifically, glucose levels were not available for all patients with diabetes, and pulmonary artery catheters were not placed in all patients. Another possible limitation was that glycosylated hemoglobin values were not available. Although glycosylated hemoglobin would more accurately reflect glucose control over a longer preoperative period of time, a single glucose level before the operation likely represents glucose control immediately preceding surgery.

The high morbidity and mortality of post-CABG DSSI detected in previous studies ${ }^{2,3,5,15}$ also was detected in our study. Consistent with other studies, 5,13 we detected a significantly longer length of stay for those patients who had a DSSI. Therefore, in addition to the catastrophic human consequences, there is significant economic impact on patients and/or hospitals as a result of post-CABG DSSI.

The human and economic impact of DSSI is a compelling reason to evaluate and change hospital policies and practices that may be contributing to DSSI. Several interventions are likely to be beneficial in preventing DSSI, and the solutions often require a multidisciplinary approach. Improved preoperative glucose control will require coordination between cardiothoracic surgeons, cardiologists, primary care physicians, and endocrinologists. Appropriate timing of antimicrobial prophylaxis requires coordination between cardiothoracic surgeons and anesthesiologists. The association of staple use for skin closure in patients with a normal BMI is intriguing and plausible; however, it requires validation in a larger study. Together, these interventions could help decrease the risk of post-CABG DSSI and reduce overall morbidity and mortality.

We acknowledge L. Douglas Cowgill, MD, and Vijay Kantamneni, MD, for their contributions during the study and preparation of the manuscript.

\section{REFERENCES}

1. American Heart Association. Heart and stroke facts 1995. Statistical supplement 1995.

2. Loop FD, Lytle BW, Cosgrove DM, et al. Sternal wound complications after isolated coronary artery bypass grafting: early and late mortality, morbidity, and cost of care. Ann Thorac Surg 1990; 49:179-86.

3. El Oakley R, Paul E, Wong PS, et al. Mediastinitis in patients undergoing cardiopulmonary bypass: risk analysis and midterm results. J Cardiovasc Surg 1998;38:595-600.

4. Breyer RH, Mills SA, Hudspeth AS, Johnston FR, Cordell AR. A prospective study of sternal wound complications. Ann Thorac Surg 1984;37:412-6.

5. Borger MA, Rao V, Weisel RD, et al. Deep sternal wound infection: risk factors and outcomes. Ann Thorac Surg 1998;65:1050-6.

6. Demmy TL, Park SB, Liebler GA, et al. Recent experience with major sternal wound complications. Ann Thorac Surg 1990;49: 458-62.

7. Kluytmans JA, Mouton JW, Maat AW, Manders MA, Michel J. 
Surveillance of postoperative infections in thoracic surgery. J Hosp Infect 1994;27:139-47.

8. Zerr KJ, Furnary AP, Grunkemeier GL, Bookin S, Kanhere V, Starr A. Glucose control lowers the risk of wound infection in diabetics after open heart operations. Ann Thorac Surg 1998;63: 356-61.

9. Grossi EA, Culliford AT, Krieger KH, et al. A survey of 77 major infectious complications of median sternotomy: a review of 7,949 consecutive operative procedures. Ann Thorac Surg 1985; 40:214-23.

10. Simchen E, Shapiro M, Marin G, Sacks T, Michel J. Risk factors for post-operative wound infection in cardiac surgery patients. Infect Control 1983;4:215-20.

11. Kouchoukos NT, Wareing TH, Murphy SF, Pelate C, Marshall WGJ. Risks of bilateral internal mammary artery bypass grafting. Ann Thorac Surg 1990;49:210-7.

12. Zacharias A, Habib RH. Factors predisposing to median sternotomy complications: deep vs superficial infection. Chest 1998; 110:1173-8.

13. Kluytmans JA, Mouton JW, Ijzerman EP, et al. Nasal carriage of Staphylococcus aureus as a major risk factor for wound infections after cardiac surgery. J Infect Dis 1995;171:216-9.

14. Ottino G, De Paulis R, Pansini S, et al. Major sternal wound infection after open-heart surgery: a multivariate analysis of risk factors in 2,579 consecutive operative procedures. Ann Thorac Surg 1987;44:173-9.

15. The Parisian Mediastinitis Study Group. Risk factors for deep sternal wound infection after sternotomy: a prospective, multicenter study. J Thorac Cardiovasc Surg 1996;111:1200-7.

16. Nishida H, Grooters RK, Soltanzadeh H, Thieman KC, Schneider
RF, Kim WP. Discriminate use of electrocautery on the median sternotomy incision: a $0.16 \%$ wound infection rate. J Thorac Cardiovasc Surg 1991;101:488-94.

17. Horan TC, Gaynes RP, Martone WJ, Jarvis WR, Emori TG. CDC definitions of nosocomial surgical site infections, 1992: a modification of CDC definitions of surgical wound infections. Infect Control Hosp Epidemiol 1992;13:606-8.

18. Kleene AR, Cullen DJ. Therapeutic Intervention Scoring System: Update 1983. Crit Care Med 1983;11:1-3.

19. Ascaso JF, Arbona C, Alcacer F, Serrano S, Hernandez A, Martinez-Valls J. Infection in diabetes: the relation to the degree of metabolic control and protein malnutrition. Med Clin 1990;95:721-4.

20. Hostetter MK. Handicaps to host defense: effects of hyperglycemia on C3 and Candida albicans. Diabetes 1990;39:271-5.

21. Alexiewicz JM, Kumar D, Smogorzewski M, Massry SG. Elevated cytosolic calcium and impaired proliferation of B lymphocytes in type II diabetes mellitus. Am J Kidney Dis 1997;30: 98-104.

22. Krolewski AS, Laffel LM, Krolewski M, Quinn M, Warram JH. Glycosylated hemoglobin and the risk of microalbuminuria in patients with insulin-dependent diabetes mellitus. N Engl J Med 1995;332:1251-5.

23. Rydall AC, Rodin GM, Olmsted MP, Devenyi RG, Daneman D. Disordered eating behavior and microvascular complications in young women with insulin-dependent diabetes mellitus. N Engl J Med 1997;336:1849-54.

24. Classen DC, Evans RS, Pestotnik SL, Horn SD, Menlove RL, Burke JP. The timing of prophylactic administration of antibiotics and the risk of surgical-wound infection. N Engl J Med 1992; 326:281-6.

\section{Targeted}

The Journal of Thoracic and Cardiovascular Surgery gives you two tables of contents.

The condensed table of contents tells you at a glance what topics and authors are presented each month. The expanded table of contents gives you a brief abstract of each article. You select only those articles of most interest to you for further reading. 